\title{
Genealogía de la práctica pedagógica para la formación de maestros normalistas ${ }^{1}$
}

\author{
María Aracelly López $\mathrm{Gil}^{2}$
}

El estudio genealógico de la práctica pedagógica para la formación de los maestros Normalistas intenta producir una ontología histórica de los modos de sujeción en relación con el campo de poder a través del cual nos construimos en sujetos que actúan sobre los demás.

La genealogía tiene como tarea específica percibir la singularidad de los sucesos encontrados en la práctica docente de la época estipulada, donde menos se esperaba en aquello que pasa desapercibido por no tener nada de historia: los sentimientos de los normalistas y de los maestros de práctica, el eros pedagógico, la conciencia del querer ser maestro y la vocación.

Atendiendo a lo anterior se presentan las formas poder y saber manifiestas en la práctica pedagógica de formación de maestros como resultado de las experiencias cotidianas en el ejercicio de la profesión docente, es decir las acciones que se hacen visibles en el encuentro con otros saberes, ciencias, creencias o prácticas en el aula.

Palabras Claves: Genealogía, poder, saber, experiencia, encuentro, práctica pedagógica, normalista.

\section{Genealogy of the pedagogical practice for the formation of the normalistas teachers}

The genealogical study of the pedagogical practice for the Normalistas Teachers formation tries to produce a historic ontology of the ways of subjection in relation to the field of power through which we build us in subjects that act on the others.

The genealogy has as specific task perceiving the singularity of the events found in the educational practice of the stipulated epoch, where except it was expected and in it that passes unnoticed by have not anything of history: the feelings of the Normalistas teachers and of the teachers of practice the pedagogical eros, the conscience of the wanted to be a teacher and the vocation.

\footnotetext{
${ }^{1}$ Recibido: Mayo 23 del 2010. Aprobado: Junio 11 del 2010.

${ }^{2}$ María Aracelly López Gil. Nacionalidad: colombiana. Docente Facultad de Educación de la Universidad de Manizales, Doctora en Ciencias de la educación RUDECOLOMBIA. Cade Universidad de Caldas. Correo Electrónico: aritalopez@hotmail.com
} 
Attending to the previous thing they are presented the form of power and know present in the pedagogical practice of formation of teachers as a consequence of the routine experiences in the exercise of the educational profession, is to say the actions that they do visible in the meeting with another know ledges, sciences ,believes or practices in the classroom .

Keywords: Genealogy, to be able, to know, experience, meeting, pedagogical practice, primary teacher.

\section{Genealogía de la práctica pedagógica.}

La genealogía de la práctica pedagógica que se trata en este artículo es una forma de mirar y escribir la historia que difiere de la historia tradicional porque se asume como historia de la práctica con perspectiva crítica e interesada en sus diferentes posibilidades de existencia. Se parte de la práctica que vivencian los normalistas hoy para hacer un recorrido a través de la urdimbre de luchas y conflictos que han configurado la práctica pedagógica de formación de maestros para preescolar y primaria tal como la conocemos y vivenciamos hoy.

Los materiales históricos que contiene la práctica, tales como las fuentes, los escritos de las diferentes épocas, lo que dicen los normalistas de ayer y maestros de hoy, no se revisan con un interés erudito, sino con el objeto de comprender cómo se gestaron las condiciones que conforman el presente de la práctica de formación de maestros normalistas. Es una mirada que toma posición por quienes reciben y son afectados de saberes y poderes específicos, propios de las relaciones de poder y de las luchas que se comprometen y tejen alrededor de la práctica.

Esta búsqueda se realiza poco a poco, a partir de la reflexión necesariamente histórica, sobre las situaciones dadas de la práctica (poderes y estrategias) y su conexión con un dominio de exterioridad.

La genealogía no implica que todas las perspectivas produzcan un mismo resultado, o que no haya criterios para jerarquizarlas, o decir cuál nos parece más justa o verdadera, sino que nos recuerda que esta decisión es un acto propio, es decir, un acto político, porque en el caso de la práctica implica tomar posición frente a una realidad de formación de maestros que ha sido envuelta en la cultura normalista que se referencia en las vivencias pedagógicas de formación de los maestros, debatidas entre la Normal, la Anexa y las escuelas afiliadas a la Normal para llevar a cabo la práctica en los contextos locales.

\section{Formas de saber y poder presentes en la experiencia de la práctica pedagógica de formación de los maestros normalistas.}


Para comprender e interpretar las formas de poder-saber presentes en la experiencia de práctica, es menester tener claro que las prácticas, por sí mismas, son "fuerzas de poder formas de saber, exterioridades, acontecimientos imposibles de prever o controlar de forma racional ... Esto quiere decir que el saber no está localizado en el interior de las disciplinas, sino en su exterior, el saber busca la línea del movimiento, mientras que la disciplina busca y encuentra el reposo, la tranquilidad de los espacios fijos... El saber es entonces lo que permite establecer puentes, caminos y encuentros con otros saberes y aun con disciplinas formalmente constituidas. ${ }^{3}$ De tal manera que el saber para el caso de la práctica es la forma, la herramienta para que el normalista entienda, conecte el conocimiento, la ciencia y el concepto; es el espacio donde lo estudiado en las disciplinas pedagógicas del plan de estudios de la normal va adquiriendo sentido, como los fundamentos y técnicas de la enseñanza, la didáctica general y las específicas, la metodología de las disciplinas, las teorías y conceptos de la pedagogía.

Lo anterior reafirma la posición de Leopold Paguay y otros, en relación a que "Los saberes prácticos son el resultado de las experiencias cotidianas de la profesión, están contextualizadas y se adquieren en una situación laboral; también los llamados saberes empíricos o de la experiencia. Los saberes sobre la práctica, pueden considerarse como procedimentales sobre cómo hacer algo y los formalizados. Los saberes de la práctica corresponden a los de la experiencia, los que resultan de una acción exitosa, de la praxis, también a los condicionales de Sternberg (1985) el saber cuándo y dónde: los distintos tipos de saber hacer. Saberes de la acción a menudo implícitos, el saber del maestro profesional, que permite distinguir al debutante del experto... Para comprender cómo se moviliza el maestro estos saberes que la mayoría de las veces no son conscientes, diversos autores han introducido el concepto operador de esquema de pensamiento, "un instrumento cognitivo" operatorio. El esquema es para Vernauud, "Io que permite que una acción sea operatoria... Malglaive (1990) nos ayuda a comprender las interacciones entre los distintos tipos de conocimiento y la acción". Entre los conocimientos teóricos, los procedimentales, los prácticos y los que se vinculan con el saber hacer, Malglaive propone el "saber en uso", que articula los diferentes conocimientos en la acción."

Los conocimientos utilizados en la práctica se convierten en nuevos saberes que J. M. Pieters, (1994) presenta como: saber declarativo es decir, ¿cómo funciona?; saber procedimental o ¿cómo hacerlo?; saber condicional, equivale a ¿cómo decidir qué hacer y cuándo? Los saberes utilizados en la acción durante el proceso de práctica van configurando el proceso de transformación de la experiencia y se construyen otros saberes "los saberes prácticos", que se dan después del proceso de adaptación del normalista al ambiente de la escuela de práctica, al grupo y a su maestro de práctica, en otras palabras, al adaptarse a la situación real de su

\footnotetext{
${ }^{3}$ Zuluaga, Olga Lucía, Echeverri Alberto y otros. 14-16 Pedagogía y Epistemología

${ }^{4}$ Paquay, Leopold, Altet Margarite, Charlier, Evelyne, Perrenoud, Philippe. "La formación profesional del maestro. Estrategias y competencias”. México. Fondo de Cultura Económico. 2005. Pág. 45-47
} 
escuela de práctica. De tal manera que el saber de la práctica se construye en la acción con la finalidad de la eficacia, es contextualizado y surge como aporte teórico cuando la acción es reflexionada.

La integración de estos saberes, para el normalista adaptarse y actuar en la situación de la práctica, permite hacer visibles unas formas de saber y poder que surgen y son transformadas en ella; éstos se han institucionalizado en la Normal, algunas de ellas se transmiten a través de los diferentes años, otras se transforman por la cultura, la reflexión, el momento social y político y otras, por el contrario, desaparecen.

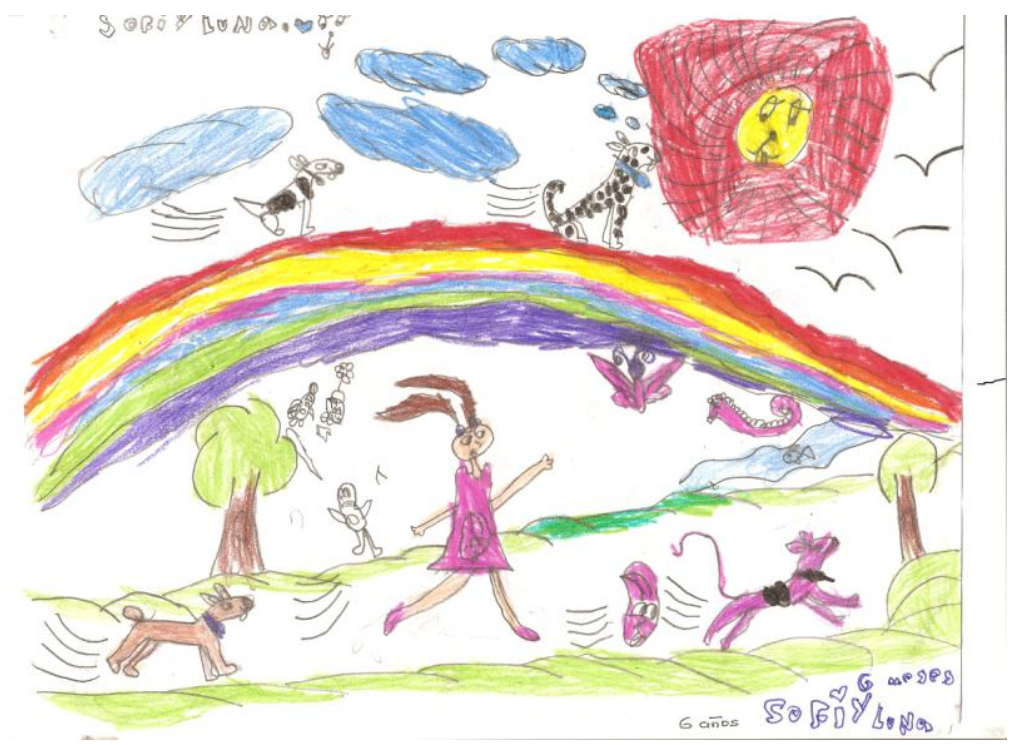

Lo anterior permite ubicar la práctica pedagógica como el espacio de ENCUENTRO con otros saberes, ciencias $\quad 0$ prácticas, que pueden generar tensiones 0 posibilidades para la creación 0 formación de otros saberes en el aula, que se dan porque el alumno maestro llega a explicar el conocer y el saber, logra explicar que reconocer es una operación de poder.

Por este anhelo de construir y probar el saber los aprendices de maestros y de otras profesiones u oficios, se sienten complacidos cuando en su proceso de formación se encuentran con el espacio de la práctica, pues es sólo a través de este encuentro donde se concretan y determinan las formas de saber y poder, que le permitirán llevar a la realidad los aprendizajes que han asimilado de las diferentes disciplinas de su formación profesional.

Ahora bien, al volver a los archivos de las prácticas pedagógicas de formación de los normalistas se acude, no para comparar o discutir o extraer verdades de ellos y adjudicarlas a las posibilidades de ejercicio de la práctica pedagógica de los maestros en ejercicio profesional, sino que acudimos a ellos para construir reflexiones claves, para lograr un acercamiento a los diversos problemas teóricos de la práctica que se realiza en el presente en las escuelas normales, que a lo largo del tiempo han configurado un campo conceptual, unos paradigmas pedagógicos y educativos que le han dado sello propio y visible a los maestros normalistas. 
En esta perspectiva, la exploración de las prácticas de los normalistas nos lleva al análisis de los saberes extraídos de los pedagogos clásicos, que mediaron la fundamentación teórica en la Normal y los avatares del concepto de enseñanza que se daba en el seno de la institución formadora de maestros.

El análisis de estas formas de saber y poder dadas en las prácticas pedagógicas de formación de los normalistas, permite identificar el pasado con el objeto de posibilitar otras maneras de ver, a partir de allí, construir otras formas de pensar la pedagogía de modo que permita una imagen de sí mismo más autónoma, libre y en relación estrecha con la otredad de su alumno y la comunidad educativa.

La formación del maestro a través de la práctica ha estado ligada a las preguntas ¿qué debe saber un maestro de primaria?, y ¿a través de qué medios es necesario formarlo? Ambas preguntas están ancladas a la "misión del maestro de preescolar y primaria" y se indagan mediante los análisis de los archivos históricos, lo dicho y lo no dicho, y se hace visible en el estudio de las diferentes formaciones discursivas realizado a la práctica en la Escuela Normal. Esta indagación patentiza unas formas de saber y poder que circulan en la práctica pedagógica de los normalistas, desde el método de la observación, experimentación y cuantificación, tales como:

- Formar en buenas costumbres, como pautas de comportamiento social y disciplinar, mediante una práctica que demuestre la formación de hábitos considerados socialmente correctos y la represión de los malos de tal manera que se creen en los niños hábitos virtuosos, modales propios de las personas bien educadas. Se visibiliza una ética que insinúa a través de sus gestos, la moral cristiana mediante un código de prohibiciones y privaciones, que en la práctica de formación prescribe, encamina o enjuicia y donde el aprendiz reproduce tomando el ejemplo de su maestro de práctica.

Tal gesto pedagógico y formativo supone preguntar: ¿ dónde se plantea una ética para el cuidado de sí mismo?, ¿cuáles son las prácticas o tecnologías para que los niños construyan y perfeccionen su mismisidad? Es decir, qué acciones puede orientar el maestro en formación para que él "con su propia fuerza o con la ayuda de otros, pueda efectuar una serie de operaciones sobre su cuerpo, su alma, su pensamiento, su comportamiento o su modo de existencia con el propósito de ir trasformándose y reconstruyendo su identidad de tal modo que consiga cierto estado de felicidad, de pureza de sabiduría, de perfección o inmortalidad" (Foucault, en Martín y otros, 1993, 26).

Tales procedimientos pedagógicos en el aula tienden un cerco disciplinario reducido a la existencia básicamente operativa, que obstaculizan pensar la pedagogía en función y relación con el conocimiento, con la sociedad que se está configurando y con la cultura. 
- Contrarrestar las influencias negativas de la socialización del niño en el medio familiar no instruido.

- Integrar el niño a la población escolar del barrio, de la localidad, de la región o del país y garantizar la aceptación del nuevo orden político, social y económico, que aparece en los diferentes niveles de socialización, orientados a obtener "orden y progreso".

Examinando estas afirmaciones es patente la influencia de los pedagogos que pensaban que el hombre o el maestro tenían que actuar sobre el niño, a través de su religión, literatura y sus costumbres, haciendo de él un ser diferente al ser natural. A esta postura Rousseau opone el ser humano educado en el contacto con la naturaleza, guiado por sus propios intereses naturales, determinado por sus capacidades y tendencias inherentes.

Para el normalista en su práctica, la orientación teórica estaba precedida de que el niño bien educado era el instruido y el culto; para Rousseau el niño bien educado era el que estaba bien desarrollado; en su obra: El Emilio" presenta el ideal de hombre racional hacia el cual debe tender la educación: el hombre de naturaleza, que supone una formación centrada exclusivamente en lo constituido de la naturaleza humana. No se educa para una profesión u oficio determinado, sino para ser hombre."5Esta ilustración muestra que en la formación de los normalistas tuvieron más influencia las concepciones pedagógicas de Pestalozzi y Herbart y demás antecesores de Rousseau.

- La habilidad para elaborar y emplear materiales gráficos y simbólicos, cuyo fin consistía en captar el interés y elevar los niveles motivacionales del grupo de niños con quienes se realizaba la práctica.

Este saber que circula en los normalistas parece que proviene de los conceptos pedagógicos de Pestalozzi y Herbart, en donde el primero, en su obra Cómo enseña Gertrudis a sus Hijos ${ }^{6}$, inicia con la intuición, la percepción directa y experimental de los objetos, captada no como meras cualidades, sino como realidad total del objeto. Forma, número y nombre, son los elementos con los cuales se describen en las preparaciones de las clases los objetos que se muestran y servirán de material real o simbólico en las respectivas prácticas.

- La preparación de las clases para la práctica y su ejecución evidencian objetivos de instrucción que pretenden en su gran mayoría la asimilación del objeto de conocimiento y tema de la clase, para lograr el éxito de la práctica pedagógica, es decir en términos de los maestros de práctica "el logro de los objetivos". Para este fin es común encontrar en los preparadores y narraciones de los normalistas de las décadas de

\footnotetext{
${ }^{5}$ Gutiérrez A, Isabel, Historia de la Educación, Madrid Ester, 1967. P. 295

${ }^{6}$ Esta obra de Pestalozzi Cómo enseña Gertrudis a sus hijos reposa en la biblioteca de la Escuela Normal Superior de Caldas y por su registro de consultas se puede estimar que tuvo marcada influencia en las estudiantes de la Normal.
} 
1960 al 1990, la planeación de una serie de pasos formales que permitían la apropiación de cualquier clase de conocimiento, y los cuales eran enseñados a los normalistas en una asignatura del área de pedagógicas denominada fundamentos o técnicas de la enseñanza; estos pasos son los que Herbart propuso en su teoría de la asimilación como principal función del espíritu y a la cual denominó apercepción: asimilación de ideas por medio de las ya adquiridas, principio fundamental de la psicología que Herbart aplicó a la educación y que se ejecuta a través de la siguiente serie de pasos aplicada y experimentada por los normalistas en sus prácticas pedagógicas:

“1) aprehensión del objeto, 2) asociación o comparación de los objetos aprehendidos, 3) etapa de sistematización o generalización que implica la comprensión de las relaciones entre los diversos objetos comparados, 4) etapa de la aplicación, en la cual la conciencia hace un esfuerzo para aplicar en sus diversas variedades el nuevo conocimiento adquirido."7

Las clases de los normalistas en su práctica eran más reconocidas y bien calificadas cuando se llevaba abundante material, no sólo real sino también simbólico, y esto era motivo de buena valoración o calificación si el material era bien utilizado por el alumno practicante; para su correcto uso se aplicaban los pasos que anteriormente se describieron y los cuales enriquecieron aquellos hábitos pedagógicos que formaron a los maestros ayer y que configuran los sujetos que hoy ejercen el magisterio .

Es de anotar que la Escuela Normal, a través de su práctica, materializa unas formas de saber relacionadas con el método de enseñanza, por una parte, y otras de manera menos profunda relacionadas con las formas de conocer, y otras a las referidas con la concepción de hombre, las cuales están en relación directa con la religión y los fines de la educación propuestos por el Estado en las diferentes reformas políticas del sistema educativo que atraviesa la práctica pedagógica de formación de los normalistas.

Las ideas de los diferentes pedagogos se enseñaban en la Normal para llevar a la práctica, a través de las diferentes unidades que conformaban su estructura y que fueron objeto de planeación en las décadas anteriores; de esta manera el sistema planteado para hacer las prácticas de los normalistas era capaz de poner una serie de estos conceptos en acción. "En el enfoque histórico que se utiliza, cuando una práctica se dota de conceptos capaces de poner en acción todo un sistema, reconocemos en ese estado de cosas el momento de una positividad, de individualización de una práctica de saber"8

Todos los saberes que se van construyendo en la práctica pedagógica de formación de normalistas giran en torno al niño, al método, a la escuela y al maestro. Se operacionalizan a través de los manuales que son el eje concentrador de todas las teorías de enseñanza que circulan en la escuela

\footnotetext{
${ }^{7}$ Zuluaga Olga Lucía, Echeverri Alberto y otros. Pedagogía y Epistemología. Pág. 56

${ }^{8}$ Zuluaga Olga Lucía, Echeverri Alberto y otros Pedagogía y epistemología. Pág 62.
} 
Normal. Es así como el arte de enseñar y aprender que caracteriza la naturaleza formadora de la Normal y que se materializa en la práctica pedagógica, es una herencia comeniana que retoman las normales vivenciando lo dicho por Comenio:" vamos a construir a manera de roca inmóvil los fundamentos de enseñar y aprender..." refiriéndose a su didáctica.

En la escuela normal circula a través de las distintas épocas la creencia que el arte de la enseñanza sólo puede adquirirse por la observación y la práctica ilustrada con la crítica pedagógica; para que un maestro posea competencia debe haber aprendido este arte en el duro yunque de la escuela de aplicación, como lo dicen Gabriela Diker y Flavia Terigi: "Un alumno libre no puede dar testimonio fehaciente de la parte más importante de la enseñanza: la práctica modelo. Puede quizá conocer la teoría de la enseñanza, leyendo cualquier libro, pero generalmente desconocerá el arte que la Escuela Normal sigue, parece también que el título de maestro normalista, quiere decir diploma otorgado a alumnos que sigan el curso correspondiente a una Escuela Normal, que se ha formado modelando día a día, normalizando sus conocimientos y la práctica de la enseñanza en una Escuela Normal, y no asistiendo por accidente y desconociendo lo que diariamente se propaga en las aulas, para formar maestros 0 profesores"...9

La práctica, además, requiere de otros tipos de saberes que se configuran en su mismo devenir y encuentros, tales como: la erudición o teoría de la enseñanza, un método, poseer los fundamentos de las materias que el niño debe adquirir, una formación científica o conocimiento de la epistemología de las disciplinas que se enseñan en la escuela primaria sobre la ciencia pedagógica que hagan de un maestro un verdadero especialista de la enseñanza. "Sólo aquel que domina profundamente aquello que va a enseñar y posee la ciencia de cómo enseñarlo podrá decidir qué, cuánto y cómo transmitir conocimiento". (La educación, año 3, N. 70, febrero de 1889).

Las normales se han caracterizado por la fortaleza para formar verdaderos maestros especialistas en la enseñanza, a través de la práctica pedagógica articulada a la erudición teórica de la misma y al saber pedagógico; antes y durante los años 1960, 1970 y 1980 la preocupación residía con mayor énfasis desde el método, y a partir de las décadas 1990 y 2000 la preocupación se centra en el dominio de los contenidos que se van a enseñar y la ciencia de cómo enseñarlos y la decisión de qué, cómo, cuánto y cuándo enseñarlo. Es un aprendizaje que avanza desde la crítica pedagógica en una práctica que se reflexiona en la cotidianidad del aula y de la escuela. A partir de la práctica se forman maestros competentes para dirigir una clase y organizar una escuela, y no sólo eruditos de un saber disciplinar, incompetentes frente a la realidad del aula y la cotidianidad escolar.

\footnotetext{
${ }^{9}$ Diker, Gabriela y Terigi, Flavia La formación de maestros y profesores: hoja de ruta. ED. Paidós Buenos Aires 2005 Pág. 40-41
} 


\section{En conclusión.}

La práctica así mismo se ha centrado en la función educativa llevándola a desarrollar una formación pedagógica asistencial, es decir centrada en el entrenamiento y cuidado de los niños, tanto en los jardines infantiles como en la misma escuela, aspecto éste aplicado en las prácticas antecedentes a las décadas del noventa y dos mil y acentuado con la práctica de comunidad en los hogares comunitarios o "práctica de PEFADI", (programa de educación familiar para el desarrollo de la infancia).

La práctica moldea cualidades personales y normaliza los conocimientos del futuro maestro; así su tarea es "forjar al maestro" y bien lo reafirma el lema de la escuela Normal Superior de Caldas propuesto desde hace más de cinco décadas: "Forjadora de maestros y pionera de la educación y cultura de Caldas"; esta tarea sólo se construye en la dura exigencia de la escuela Anexa o afiliadas a la Normal, cuyo énfasis es normalizador/disciplinador, no sólo de las cualidades personales sino también de los conocimientos.

El balance disciplina/pedagogía en la formación de profesores conforma un debate de la etapa fundacional de la práctica docente. De esta forma la escuela Normal va configurando un sujeto que siente y vive la necesidad de mantenerse actualizado respecto a las "conquistas de la pedagogía"; en este mismo proceso de configuración del maestro, la Normal conecta su formación con la creciente heterogeneidad social y cultural de la población, con la cual habrá de sostener la relación educativa; de esta manera la práctica se sitúa en diversos contextos y grupos de estudiantes, porque la misión actual de la Normal de Caldas desde su propuesta pedagógica problémica reconoce las diversidades sociales y sus problemas, los cuales requieren marcos conceptuales concretos y fundamentalmente en la práctica, estrategias de acción para abordar e incluir en los encuentros pedagógicos de su escuela el problema de la violencia con sus consecuencias y connotaciones (los reinsertados, desplazados, paramilitares...). Dichas poblaciones consideradas vulnerables son cada vez más frecuentes en las escuelas y deben ser asumidas por el maestro en formación, de donde el ámbito de decisión para tratar la diversidad social termina siendo el trabajo o práctica pedagógica, porque las estrategias espontáneamente desarrolladas por los practicantes en ejercicio, cuando se encuentran con estas situaciones, son revestidas de carácter proactivo y de la gran probidad que le otorga su utilidad inmediata; de allí que terminan siendo naturalizadas como saber profesional legítimo.

Se puede decir que los saberes que engendra la práctica se centran en aquellos que se convocan en la enseñanza como campo conceptual y operativo del saber pedagógico en la Normal. La inserción del normalista en el mundo escolar y sus fuertes vínculos con el trabajo comunitario ha permitido configurar un sujeto social y trabajador de la cultura, que en su trayecto de formación va asumiendo la pedagogía como puente de 
encuentro entre el hombre, su lenguaje, el conocimiento y las interioridades de la cultura con que se relaciona.

Los sistemas de acción colectiva que tejen la cotidianidad de la formación de maestros normalistas configuran sujetos con sentido de niñez, pueblo, comunidad, ruralidad, cuyo eros social los lleva por los diferentes contextos de la patria entregando sus saberes artísticos, formales y procedimentales que han tenido enraizamiento en la filosofía de la ilustración a partir del concepto de formación del hombre con su paquete axiológico, ético, moral y religioso el cual se va mezclando desde la década del sesenta con las interacciones, tanto en su interior como en su exterior con las ciencias de la educación.

\section{Bibliografía}

- Albano, Sergio. Michel Foucault, (2006). Glosario de aplicaciones. Buenos Aires, Argentina. Quadrata imaginarios. Bogotá Colombia. Logoformas S.A.

- Archivo Historial de la Escuela Normal Superior de Manizales (Caldas).

- De Tezanos. Araceli. (2006). El maestro y su formación, tras las huellas y los imaginarios. Bogotá Colombia. Logoformas S.A.

- Diker Gabriela y otro. (2005). La Formación de maestros y profesores: hoja de ruta. Buenos Aires: Paidos.

- Diker, Gabriela y otros. (2005). La Formación de maestros y profesores: hoja de ruta. Buenos Aires: Paidos.

- Dilthey, Guillermo. (1944). Historia de la Pedagogía. Argentina. Losada

- Dussel, Inés y otro. (1999). La invención del aula. Buenos Aires. Ed. Santillana.

- Escudero, Juan Manuel y otros. (2006). La formación del profesorado y la mejor educación, políticas y prácticas. España, Ed. Octaedro.

- Ferry Gilles. (1991). El trayecto de la formación, los enseñantes entre la teoría y la práctica. Barcelona: Ed. Paidos. 
- Foucault, Michel. (2004). Nietzsche, la genealogía, la historia Valencia, España. Pre-textos.

- (2005). Glosario de aplicaciones; Buenos Aires:

Ed. Quadrata.

(1970). La arqueología del saber; Buenos Aires: siglo XXI editores.

(1981). Un diálogo sobre el poder y otras conversaciones; Madrid: Alianza Editorial, S.A., y Materiales, S.A., de estudios y publicaciones.

y otros. (1991). Espacios de poder. Madrid, España. La piqueta.

.. (1998). Las palabras y las cosas. Madrid. Siglo veintiuno.

$\bullet$ (2006). Seguridad, territorio, población. Buenos Aires, Argentina. Fondo de cultura económica.

.. (2005). Memorias seminario. Bogotá Colombia.

Ed. Uní ediciones.

(2005). La Verdad y las formas Jurídicas. Argentina. Ed. Gedisa.

- Larrosa, Jorge. Escuela, Poder y Subjetividad. Madrid. Ed. La piqueta.

- Quiceno C., Humberto. (2003). Crónicas Históricas de la Educación en Colombia. Bogotá Colombia. Delfín, Ltda.

- Rodríguez Rojas, José M, (1961). Pedagogía y metodología general. Medellín Colombia, Ed. Bedout.

- Zuluaga G. Olga Lucía, ECHEVERRI S. Alberto, MARTINEZ B. Alberto, QUICENO C. Humberto, SAENZ O. Javier, ALVAREZ G. Alejandro. (2003). Pedagogía y epistemología; Bogotá (Colombia): Ed. Delfín, Ltda.

(2005). Foucault, la pedagogía y la educación. pensar de otro modo; Bogotá (Colombia): Ed. Delfín, Ltda. 